\title{
Flow characteristics of liquid with pressure-dependent viscosities in microtubes
}

Received: 28 September 2004 / Revised: 11 July 2005 / Accepted: 2 November 2005 / Published online: 10 January 2006 (C) Springer-Verlag 2006

\begin{abstract}
It is obvious that the pressure gradient along the axial direction in a pipe flow keeps constant according to the Hagen-Poiseuille equation. However, recent experiments indicated that the distribution of the pressure seemed no longer linear for liquid flows in microtubes driven by high pressure (1-30MPa). Based on H-P equation with slip boundary condition and Bridgman's relation of viscosity vs. static pressure, the nonlinear distribution of pressure along the axial direction is analyzed in this paper. The revised standard Poiseuille number with the effect of pressure-dependent viscosity taken into account agrees well with the experimental results. Therefore, the dependence of the viscosity on the pressure is one of the dominating factors under high driven pressure, and is represented by an important property coefficient $\alpha$ of the liquid.
\end{abstract}

Keywords Microfluidic $\cdot$ Liquid $\cdot$ Microtube $\cdot$ Viscosity

\section{Introduction}

The characteristics of liquid flows in microchannels are important in designing microfluidic devices. Pfahler et al. [1] measured the friction factor of isopropanol in a microchannel with a hydraulic diameter of $39.7 \mu \mathrm{m}$, and found that its apparent viscosity became smaller than that in macro-

The project supported by the Chinese Academy of Sciences Major Innovation Project (KJCX2-SW-L2) and the National Natural Science Foundation of China (10272107).

The English text was polished by Yunming Chen.

Z.H. Silber-Li $(\varangle) \cdot$ H.H. Cui · Y.P. Tan

LNM, Institute of Mechanics, CAS, Beijing, 100080, China

E-mail: lili@imech.ac.cn

Tel.: 010-62524839

Fax: 010-62579511

Y.P. Tan

SIAMM, Shanghai University, Shanghai 200072, China

P. Tabeling

MMN, ESPCI, 10 rue Vauquelin, Paris, 75231, France scale cases, whereas Papautsky's [2] experiments with water (Reynolds number $=0.001-18$ and hydraulic diameter $=$ $57 \mu \mathrm{m})$ revealed that the experimental flow rate was smaller than the prediction by the classical theory. However, with more accurate measurement methods, the recent results of Sharp et al. [3], Li et al. [4,5] and Judy et al. [6] all indicated that the behaviors of liquid flows in microchannels with diameters larger than tens micrometer are in good agreement with the continuum theory. In order to extend the experimental research to smaller microtubes, Cui et al. [7] measured the flow characteristics of liquids in microtubes with diameters from $3 \mu \mathrm{m}$ to $10 \mu \mathrm{m}$. As the flow rate is proportional to the fourth power of the diameter, a higher pressure (1-30 MPa) must be applied to maintain a flow rate that can be accurately measured. From their experiments, they found that the viscosity of liquid along the axial direction was changed, and the pressure gradient of liquid flow seemed to be nonlinear. They gave a simplification formula of normalized friction coefficient to correlate their experimental results. However, the pressure and viscosity distributions are worth making further investigation.

In this paper, the pressure distribution along the axial direction in microtubes under a high pressure is analyzed, and the variation of viscosity along the flow direction is expressed as a function of pressure. Considering the high-pressure effect, a revised standard Poiseuille number is proposed and compared with the experimental results.

\section{Theoretical analysis}

\subsection{Velocity and flow rate}

For a steady, fully developed laminar flow in a circular crosssection tube, H-P equation in $\Delta x$ element can be expressed as

$\frac{\partial p}{\partial x}=\frac{\mu}{r} \frac{\partial}{\partial r}\left(r \frac{\partial u}{\partial r}\right)$,

where, $p$ is the pressure, $\mu$ is the viscosity, $u$ is the velocity, $x$ and $r$ are the axial and radial coordinates, respectively (Fig.1). 


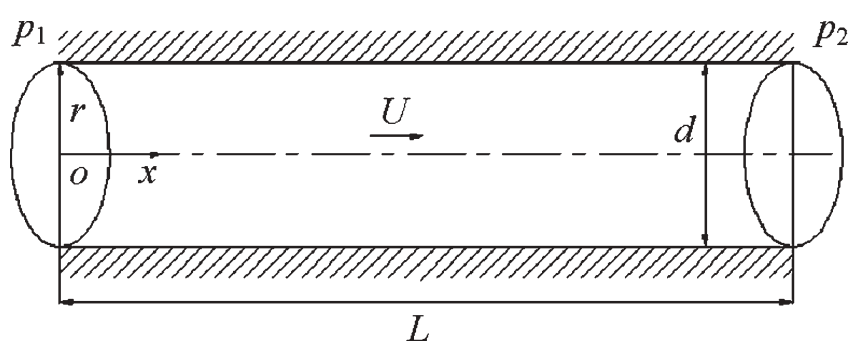

Fig. 1 A schematic depiction of the flow in microtubes

Based on the experiments of Choi et al. [8] and Tretheway et al. [9] in microchannels, the slip boundary condition should be considered,

$r=\frac{d}{2}, \quad u=-L_{s} \dot{\gamma}_{W}=-L_{s}\left(\frac{\partial u}{\partial r}\right)_{W} ;$

$r=0, \quad \frac{\partial u}{\partial r}=0$,

where $L_{s}$ is the slip length, $\dot{\gamma}_{W}$ is the shear rate at wall, $d$ is the diameter of the tube.

Considering $\mu$ is not a function of $r$, because there is no effect in this direction, we integrate Eq.(1) respect to $r$ two times and get

$u(r)=\frac{1}{4 \mu}\left[r^{2}-\frac{d^{2}}{4}\right] \frac{\partial p}{\partial x}-\frac{L_{s} d}{4 \mu} \frac{\partial p}{\partial x}$.

It is easy to determine the volume flow rate $Q$ through the tube,

$$
\begin{aligned}
Q & =\int_{0}^{2 \pi} \int_{-\frac{d}{2}}^{\frac{d}{2}} u(r) \cdot r \mathrm{~d} \theta \mathrm{d} r \\
& =-\frac{\pi d^{4}}{128 \mu} \frac{\partial p}{\partial x}-\frac{\pi d^{3} L_{s}}{16 \mu} \frac{\partial p}{\partial x} \\
& =-\frac{\pi d^{4}}{128 \mu}\left(1+\frac{8 L_{s}}{d}\right) \frac{\partial p}{\partial x} .
\end{aligned}
$$

Under no slip condition, Eq.(3) and Eq.(4) are converted into the well-known H-P equation. $u(r)$ and $Q_{\text {th }}$ are expressed as

$$
\begin{aligned}
u(r) & =\frac{1}{4 \mu}\left[r^{2}-\frac{d^{2}}{4}\right] \frac{\partial p}{\partial x}, \\
Q_{\text {th }} & =\frac{\pi d^{4}}{128 \mu_{0} L} \Delta p,
\end{aligned}
$$

where $\Delta p=p_{1}-p_{2}$ is the pressure drop between the entrance pressure $p_{1}$ and the exit pressure $p_{2}, \mu_{0}$ is the viscosity under the atmospheric temperature and $L$ is the length of the tube.

\subsection{Pressure and viscosity distributions at high pressure}

According to Bridgman's results [10], the relationship of the viscosity vs. the static pressure can be expressed as an exponential function,

$\mu=\mu_{0} \mathrm{e}^{\alpha\left(p-p_{0}\right)}$, where, $\mu$ is the real viscosity, $p_{0}$ is the atmospheric pressure and $\alpha$ is a property coefficient of the liquid. Substituting Eq.(7) into Eq.(4), we obtain

$\mathrm{e}^{-\alpha p} \mathrm{~d} p=-\frac{128 \mu_{0} Q}{\pi d^{4} A} \mathrm{~d} x$

where $A=1+8 L_{s} / d$. Integrating Eq.(8) along $x$-direction, we get

$\int_{p_{1}}^{p} \mathrm{e}^{-\alpha p} \mathrm{~d} p=-\int_{0}^{x} \frac{128 \mu_{0} Q}{\pi d^{4} A} \mathrm{~d} x$,
$p(x)=-\frac{1}{\alpha} \ln \left[\frac{128 \mu_{0} \alpha Q}{\pi d^{4} A} x+\mathrm{e}^{-\alpha p_{1}}\right]$.

Obviously, the distribution of $p(x)$ along $x$-direction is a logarithmic function, which depends on the inlet pressure $p_{1}$ and the position $x$. It's well known that the pressure gradient along the axial direction of tube keeps constant in a classical H-P flow, therefore, the flow driven by high pressure is no longer a H-P flow.

The pressure at the exit $p_{2}$ is equal to $p_{0}$, Eq.(9) can be rewritten as

$\frac{p(x)}{p_{1}}=-\frac{\ln \left(\left(1-\mathrm{e}^{-\alpha p_{1}}\right) \frac{x}{L}+\mathrm{e}^{-\alpha p_{1}}\right)}{\alpha p_{1}}$.

Figure 2 shows the distribution of $p(x) / p_{1}$ along $x$-direction with $\alpha=7.9 \times 10^{-3} \mathrm{MPa}^{-1}$ for isopropanol.

The difference between the nonlinear pressure distribution $p(x)$ and corresponding parameters of the classical H-P flow $p_{\text {th }}(x)$ is defined as $\varepsilon\left(\varepsilon=p(x)-p_{\text {th }}(x)\right)$. Figure 3 shows the variation of $\varepsilon$ with $x / L$. The maximum value of $\varepsilon$ can reach $3 \%$.

According to Taylor formula, Eq.(7) is expanded at the atmospheric pressure $p_{0}$

$\mu(p)=\mu_{0}\left(p_{0}\right)+\left(p-p_{0}\right) \frac{\partial \mu\left(p_{0}\right)}{\partial p}+\cdots$,

where $\frac{\partial \mu\left(p_{0}\right)}{\partial p}=\left.\mu_{0} \alpha \mathrm{e}^{\alpha\left(p-p_{0}\right)}\right|_{p=p_{0}}=\mu_{0} \alpha$, we obtain,

$\mu(p) \approx \mu_{0}\left(p_{0}\right)\left[1+\alpha\left(p-p_{0}\right)\right] \approx \mu_{0}\left(p_{0}\right)[1+\alpha p]$.

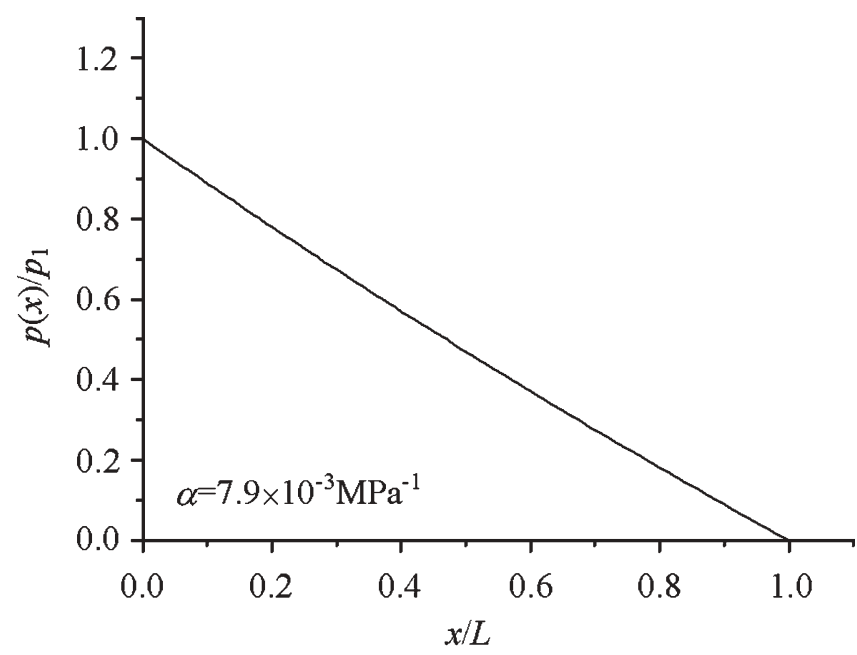

Fig. 2 Dimensionless pressure distribution along $x$-direction 


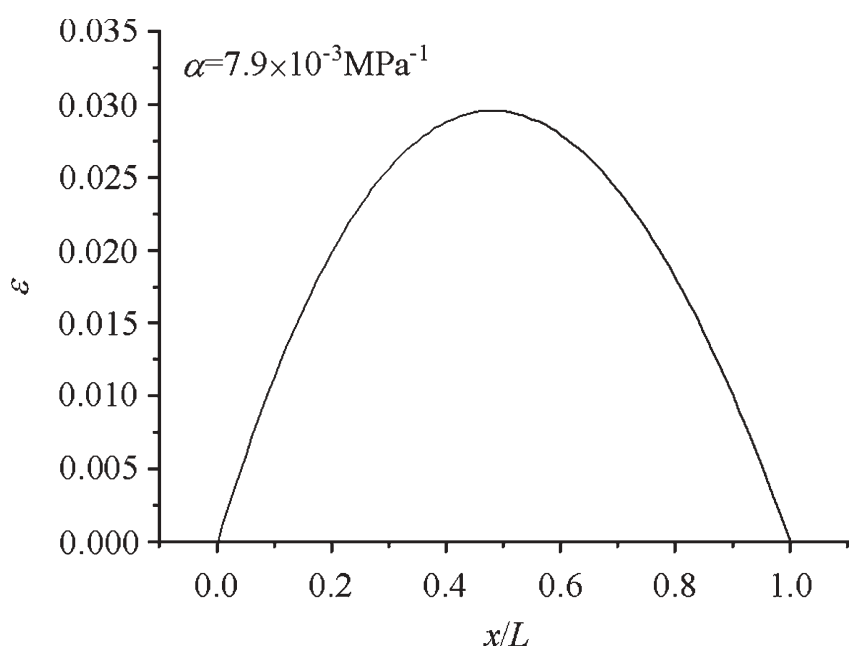

Fig. 3 Variation of $\varepsilon$ with $x / L$

Substituting Eq.(9) into Eq.(12), we noted that $\mu(p)$ is a nonlinear function along the flow direction.

\section{Comparison with experimental results}

\subsection{Experimental methods}

The experiments have been carried out in LNM laboratory [7]. The experimental setup [4,5] includes three units: a pressure source, a testing section and a flux-measuring unit (Fig.4). The high-pressure pump of 1-40MPa (147-5800Psi) is used to drive the working liquids. The testing section consists of a tee connector, a pressure transducer, a thermocouple and a microtube. The pressure transducer and the thermocouple are connected to the tee connector, through which the inlet pressure of the microtube and the temperature of the working liquid can be measured. The microtube is long enough to neglect the inlet and outlet effects $(d=3-10 \mu \mathrm{m}, L \approx 50 \mathrm{~mm})$. One end of the microtube is linked to the tee connector with a mechanical seal and the other end is inserted into a glass capillary with glues. The working liquid passing through the microtube is gathered in the glass capillary. Through measuring the displacement $s$ of liquid's column surface in the capillary and the interval $t$, the flow rate $Q_{\exp }$ can be calculated by the following equation:

$Q_{\exp }=\frac{\pi D^{2} s}{4 t}$

where $D$ is the diameter of the capillary. The test liquid presented here is only isopropanol.

The inner surface roughness of microtube is measured with AFM. The average height $\Delta h$ is about $70-7.12 \mathrm{~nm}$. The relative surface roughness is calculated to be less than $0.7 \%$. So the microtube can be regarded as the hydraulic smooth tube. The influences of surface evaporation, viscous heating and liquid's compressibility etc. can be neglected in this experiment [7].

The experimental uncertainty consists of two parts: the uncertainty of the experimental system and a product of the standard deviation and $\zeta$, where $\zeta$ is a coefficient related to the number of measurements. The uncertainty of experimental system mainly depends on the precision of the diameter measurement [7]. With images of SEM, the measurement precision is about $\pm 0.1 \mu \mathrm{m}$. Therefore, the measurement uncertainties are $\pm 1 \%- \pm 3.3 \%$ for tubes of $3-10 \mu \mathrm{m}$ diameters. Because the flow rate is proportional to the forth power of the diameter, the experimental uncertainties for three microtubes $(10 \mu \mathrm{m}, 5 \mu \mathrm{m} 3 \mu \mathrm{m})$ are $\pm 4.8 \%, \pm 8.6 \%$ and $\pm 13.6 \%$,

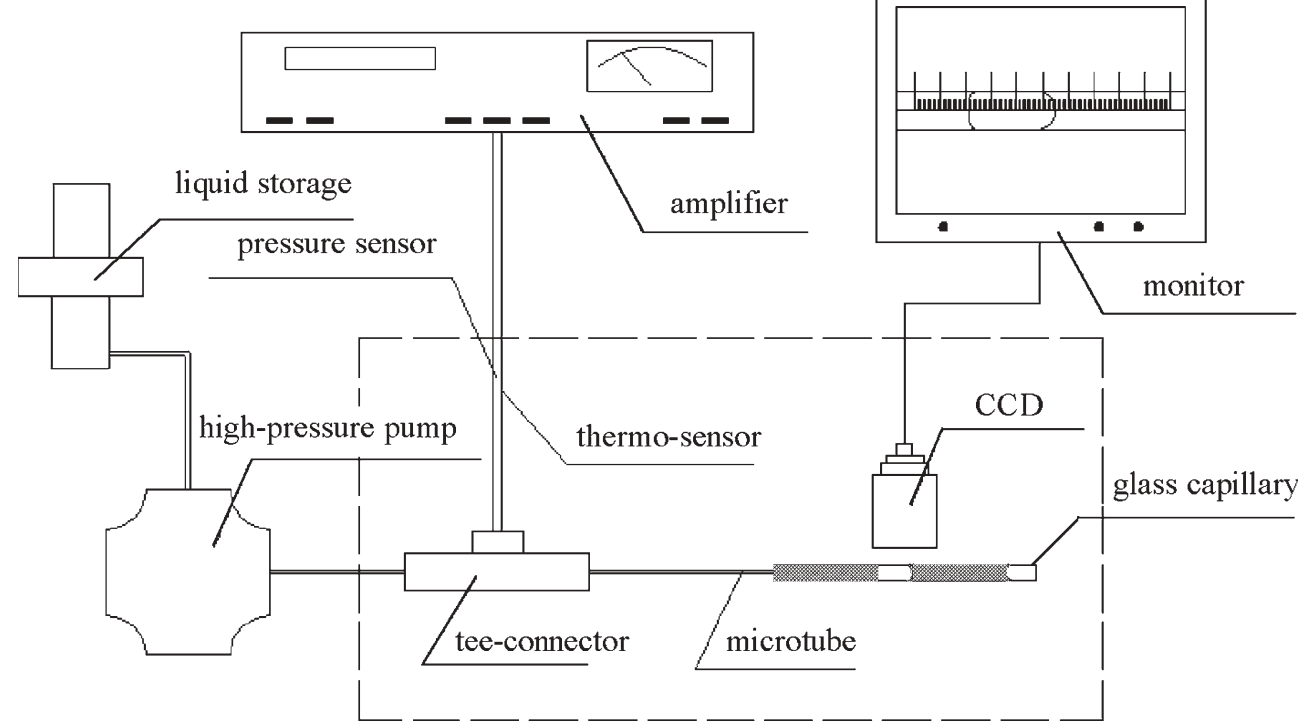

Fig. 4 A sketch of the high-pressure microflow setup 
respectively. The experimental results are presented as individual arithmetic average values with corresponding experimental error bars. Each arithmetic average value is calculated from at least three measurements.

\subsection{Experimental results}

Poiseuille Number $P o$ in a pipe flow is defined as $P o=f R e$, $f$ is the Fanning friction coefficient [11]. $P o=16$ for the flow in a tube with a circular cross-section. To compare the theoretical values with the experimental results, the standard Poiseuille Number $P o^{*}$ is defined as

$P o^{*}=\frac{P o_{\mathrm{exp}}}{P o_{\mathrm{th}}}=\frac{(\operatorname{Re} \cdot f)_{\mathrm{exp}}}{(\operatorname{Re} \cdot f)_{\mathrm{th}}}=\frac{Q_{\mathrm{th}}}{Q_{\mathrm{exp}}}$,

where the subscripts th and exp present the theoretical and experimental values, respectively. If the value of $Q_{\text {exp }}$ agrees with the prediction by H-P equation, $P o^{*}=1$. However, from the experimental results, $P o^{*}$ deviates from 1 and the values increase noticeably with the pressure (Fig.5-7). Even though the uncertainty is considered, experimental results are still 3\%-5\% larger than 1 at the pressure of 30MPa (Fig.5).

\subsection{Revised standard poiseuille number $P o^{* \prime}$}

Considering the dependence of the viscosity on the pressure, the revised $P o^{* \prime}$ is defined as

$P o^{* \prime}=\frac{P o_{\mathrm{th}-\mu}}{P o_{\mathrm{th}}}=\frac{Q_{\mathrm{th}}}{Q_{\mathrm{th}-\mu}}$,

where $Q_{\mathrm{th}-\mu}$ is the flow rate when the revised viscosity is taken into account. From Eq.(9), $Q_{\mathrm{th}-\mu}$ is given as

$Q_{\mathrm{th}-\mu}=\frac{\pi d^{4} A}{128 \mu_{0} \alpha L} \mathrm{e}^{-\alpha p_{1}}\left(\mathrm{e}^{\alpha \Delta p}-1\right)$.

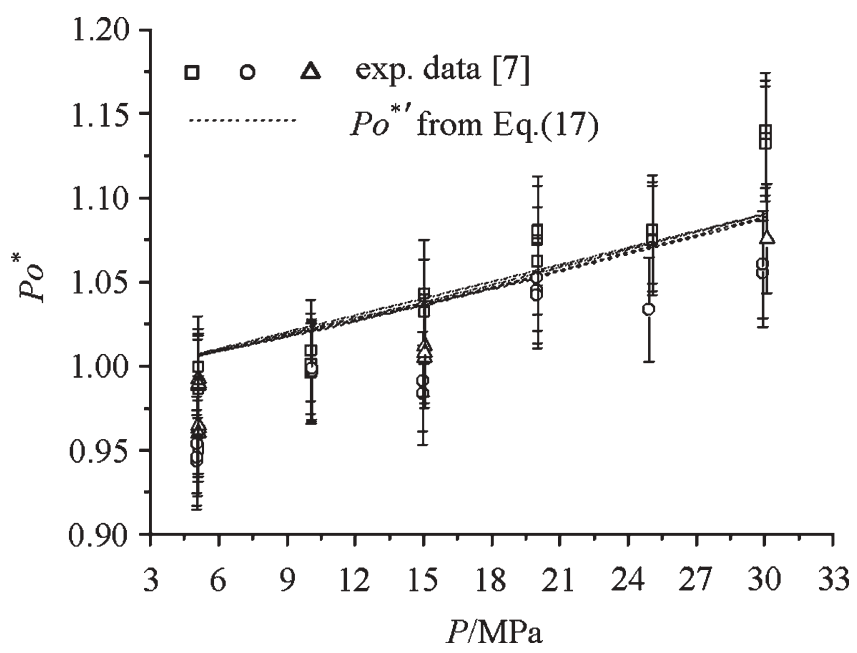

Fig. 5 Comparison of $P o^{* \prime}$ vs. the pressure drop of isopropanol in $10 \mu \mathrm{m}$ tube

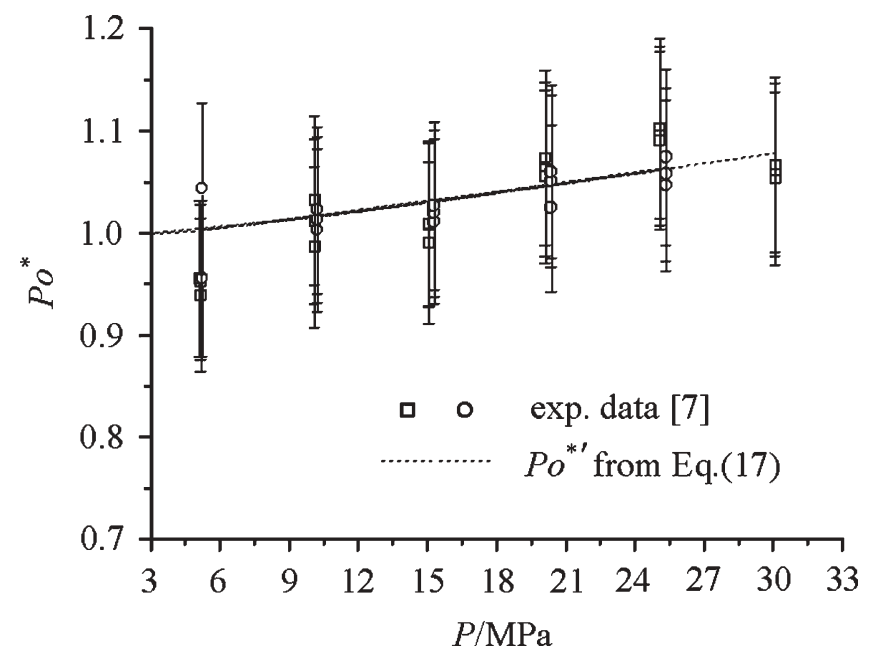

Fig. 6 Comparison of $\mathrm{Po}^{* \prime}$ vs. the pressure drop of isopropanol in $5 \mu \mathrm{m}$ tube

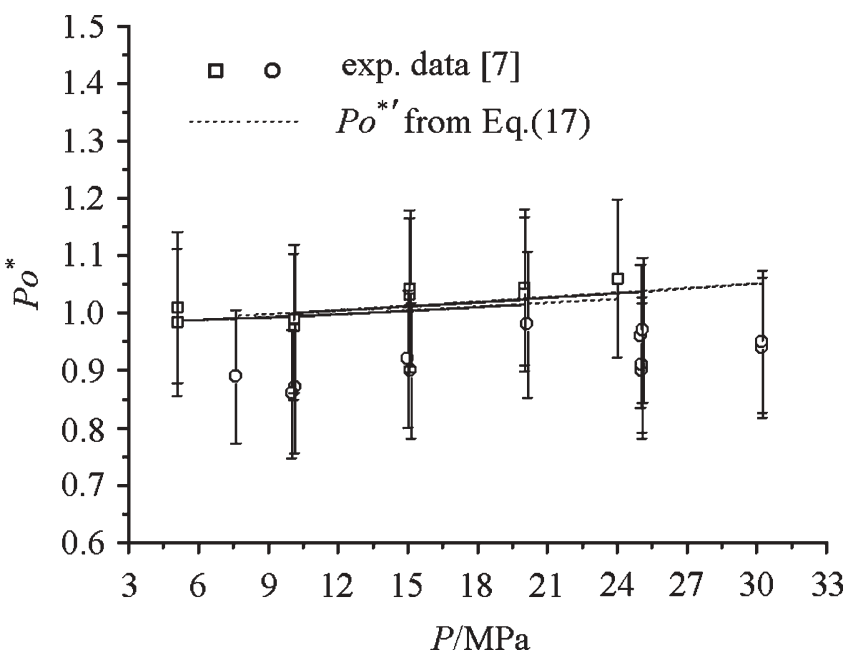

Fig. 7 Comparison of $P o^{* \prime}$ vs. the pressure drop of isopropanol in $3 \mu \mathrm{m}$ tube

The revised standard Poiseuille number $P o^{* \prime}$ is

$$
\begin{aligned}
P o^{* \prime} & =\frac{Q_{\mathrm{th}}}{Q_{\mathrm{th}-\mu}} \\
& =\mathrm{e}^{\alpha p_{1}} \cdot \Delta p\left(\mathrm{e}^{\alpha \Delta p}-1\right)^{-1} \cdot \frac{\alpha}{A}=\frac{\alpha \Delta p \mathrm{e}^{\alpha p_{1}}}{A \cdot \mathrm{e}^{\alpha \Delta p}-1} .
\end{aligned}
$$

The increment of the average flow rate resulted from the slip condition is smaller than $5 \%$ at $30 \mathrm{MPa}$ in the case of 10 $\mu \mathrm{m}$ microtube [7], so $A$ is equal to 1.05. In Fig.5, the revised standard Poiseuille number $P o^{* \prime}$ is presented as a dash line. It increases significantly with the pressure and shows the same trend as the experimental results. This reveals that the revised flow rate agrees well with the experimental value. It means that the dominant influence on the viscosity is the high pressure. The same trends appear in the microtubes with the diameter of $3 \mu \mathrm{m}$ and $5 \mu \mathrm{m}$, respectively. 


\subsection{Discussions}

(1) Based on Eq.(9), the nonlinear distribution of pressure depends on the property of the liquids, i.e. the coefficient $\alpha$. For most liquids, except for water, the values of $\alpha$ are nearly $10^{-2}-10^{-3} \mathrm{MPa}^{-1}[10]$. When the driven pressure becomes higher than $10 \mathrm{MPa}$, the effect of pressuredependent viscosity should be considered. This is confirmed by experimental results. In Fig. $5, P o^{*}$ is noticeably larger than 1 when $p>10 \mathrm{MPa}$;

(2) For water, because of hydrogen bond, the value of $\alpha$ keeps near zero when the driven pressure is smaller than $100 \mathrm{MPa}$ [10]. The experimental results of Cui et al. [7] also demonstrated that the viscosity of water in microflows keeps constant for pressures ranging from 1-30MPa.

\section{Conclusions}

When the diameters of microtubes are smaller than $10 \mu \mathrm{m}$, the driven pressure should be increased to 1-30MPa. The high pressure leads to variation in the viscosity of liquids. In this paper, we analyzed both the pressure distributions and the viscosity distributions along the flow direction, and introduced a revised standard Poiseuille number to correlate the experimental results. The main results are as follows:

(1) The distributions of the pressure and the viscosity become nonlinear along the axial direction. The behavior of the nonlinearity is mainly related to the property coefficient of the liquid $\alpha$. For example of isopropanol, the difference between the nonlinear pressure distribution and the corresponding parameters of classical H-P flow reaches $3 \%$;

(2) The revised standard Poiseuille number $P o^{* \prime}$ agrees well with the experimental results. It means that the dependence of the viscosity on the pressure is a dominant factor in microflows under high pressure;

(3) The variation of viscosity under high pressure dominantly depends on the property coefficient $\alpha$ of the liquid. For most liquids, except for water, the effect of pressuredependent viscosity should be considered if the driven pressure is higher than $10 \mathrm{MPa}$.

\section{References}

1. Pfahler J., Harley J., Bau H., Zemel J.: Gas and liquid flow in small channels. Am Soc Mech Eng Dynam Syst Control Division 32, 49-60 (1991)

2. Papautsky I., Brazzle J., Ameel T., Frazier A.B.: Laminar fluid behavior in microchannels using micropolar fluid theory. Sensors and Actuators 73, 101-108 (1999)

3. Sharp K.V., Adrian R.J.: Transition from laminar to turbulent flow in liquid Filled Microtubes. Experiments in fluids 36, 741-747 (2004)

4. Li Z.H., Zhou X.B., Zhu S.N.: Flow characteristics of non-polar organic with small molecules in a microchannel. Acta Mechanica Sinica, 34(3), 432-439 (2002) (in Chinese)

5. Li Z.H., Cui H.H.: Proceeding of experiments about liquid flow through micro-tubes. Int J Nonlinear Science Numerical Simulation 3-4, 577-580 (2002)

6. Judy J., Maynes D., Webb B.W.: Characterization of frictional pressure drop for liquid flows through microchannels. Int $\mathrm{J}$ Heat and Mass transfer 45, 3477-3489 (2002)

7. Cui H.H., Silber-Li Z.H., Zhu S.N.: Flow characteristics of liquids in microtubes driven by a high pressure. Physics of Fluids, 16(5), 1803-1810 (2004)

8. Choi C.H., Johan K., Westin A., Breuera K.S.: Apparent slip flows in hydrophilic and hydrophobic microchannels. Physics of Fluids, 15 (10), 2898-2902 (2003)

9. Tretheway D., Meinhart C.: Apparent fluid slip at hydrophobic microchannel walls. Physics of Fluids 14(3), 9-12 (2002)

10. Bridgman P.W.: The physics of high pressure. G. Bell and Sons Ltd., 1952

11. White F.M.: Viscous fluid flow. 2nd ed. McGraw Hill, 1991 